\title{
Medical school "banter" denigrating GPs and psychiatrists must stop, say college heads
}

In the print version of this story (issue dated 8 October; $B M J$ 2016;354:i5316, doi:10.1136/bmj.i5316) we used a photograph of a communal student area which we captioned as being Imperial College London. We have been informed by Imperial
College that this is not a part of its campus and apologise for the error.

We also did not want to suggest that the "banter" among students and staff referred to in the story was something that occurred at Imperial College. Again, we apologise for this inaccuracy. 